\title{
Correlates of poor medication adherence in chronic psychotic disorders
}

Martha Sajatovic, Jessie Mbwambo, Isaac Lema, Carol Blixen, Michelle E. Aebi, Betsy Wilson, Godwin Njiro, Christopher J. Burant, Kristin A. Cassidy, Jennifer B. Levin and Sylvia Kaaya

\section{Background}

Chronic psychotic disorders (CPDS) occur worldwide and cause significant burden. Poor medication adherence is pervasive, but has not been well studied in sub-Saharan Africa.

\section{Aims}

This cross-sectional survey of 100 poorly adherent Tanzanian patients with CPD characterised clinical features associated with poor adherence.

\section{Method}

Descriptive statistics characterised demographic and clinical variables, including barriers to adherence, adherence behaviours and attitudes, and psychiatric symptoms. Measures included the Tablets Routine Questionnaire, Drug Attitudes Inventory, the Brief Psychiatric Rating Scale, the Clinical Global Impressions scale, the Alcohol Use Disorders Identification Test and Alcohol, Smoking and Substance Involvement Screening Test. The relationship between adherence and other clinical variables was evaluated.

\section{Results}

Mean age was 35.7 years (s.d. 8.8), 61\% were male and $80 \%$ had schizophrenia, with a mean age at onset of 22.4 (s.d. 7.6) years Mean proportion of missed CPD medication was $64 \%$. One in ten had alcohol dependence. Most individuals had multiple adherence barriers. Most clinical variables were not significantly associated with the Tablets Routine Questionnaire; however, inpatients with CPD were more likely to have worse adherence $(P \leq 0.01)$, as were individuals with worse medication attitudes (Drug Attitudes Inventory, $P<0.01$ ), higher CPD symptom severity levels (Brief Psychiatric Rating Scale, $P<0.001$ ) and higher-risk use of alcohol (Alcohol Use Disorders Identification Test, $P<0.001$ )

\section{Conclusions}

Poorly adherent patients had multiple barriers to adherence, including poor attitudes toward medication and treatment, high illness acuity and substance use comorbidity. Treatments need to address adherence barriers, and consider family supports and challenges from an intergenerational perspective.

\section{Keywords}

Antipsychotics; psychosis; schizophrenia; medication; treatment adherence.

\section{Copyright and usage}

(c) The Author(s), 2020. Published by Cambridge University Press on behalf of the Royal College of Psychiatrists. This is an Open Access article, distributed under the terms of the Creative Commons Attribution licence (http://creativecommons.org/ licenses/by/4.0/), which permits unrestricted re-use, distribution, and reproduction in any medium, provided the original work is properly cited.
Chronic psychotic disorders (CPDs), such as schizophrenia and schizoaffective disorder, occur worldwide and cause significant burden, characterised by reduced quality of life, functional impairment and premature mortality owing to suicide and other causes. Although the standard of care for CPD includes antipsychotic drugs, poor medication adherence is pervasive globally and leads to negative outcomes that affect not just individuals with schizophrenia, but also families and communities. ${ }^{1,2}$ There are many barriers to medication-taking generally among people with schizophrenia, including erratic medication-taking, poor insight into illness, cognitive problems, stigma and limited access to care $^{1,3-5}$ A recent systematic literature review and meta-analysis of the global literature on medication non-adherence in major psychiatric disorder suggests that poor adherence is influenced by a variety of factors, such as patients' individual behaviours; social or family support; clinical, illness and/or treatment factors, and overall healthcare system-related factors. ${ }^{6}$

In sub-Saharan Africa, poor adherence is seen in approximately half of individuals with CPD; however, clinical correlates of poor adherence have not been well studied. ${ }^{7-11}$ A qualitative study from Tanzania $^{10}$ suggested that both patients and caregivers perceived non-adherence to antipsychotic medication as a leading factor of relapse. A literature review on antipsychotic treatment for people with schizophrenia in sub-Saharan Africa, by Chidarikire et al, ${ }^{1}$ noted that limited access to care, a poor understanding of illness and treatment, limited insight, lower levels of literacy, comorbid substance misuse, the presence of CPD symptoms and medication treatment side-effects can all negatively affect adherence.

To develop and deliver care approaches that may promote improved adherence, it is important to understand the clinical features and adherence barriers for people at greatest risk of poor adherence. This cross-sectional survey of patients with CPD in Tanzania characterised demographic and clinical features that appear to be associated with suboptimal medication adherence.

\section{Method}

\section{Overview}

Data from this analysis is derived from a larger 24-month project, funded by the USA's National Institute of Mental Health, to refine and preliminarily test an intervention intended to promote medication adherence in poorly adherent patients. Methods of the larger project have been described elsewhere. ${ }^{12}$ This analysis assessed clinical features and adherence correlates among 100 poorly adherent patients with $\mathrm{CPD}$ who were receiving care in the psychiatry department at a 70-bed national referral hospital located in urban Dar es Salaam, Tanzania. Demographic features, past resource use, substance use comorbidity and psychosocial support were evaluated with respect to their association with selfreported medication treatment adherence. 


\section{Study setting and recruitment}

Adults aged $\geq 18$ years, with a clinical diagnosis of schizophrenia or schizoaffective disorder, were recruited from the national psychiatric referral hospital and associated hospital ambulatory clinics. Patients at the hospital are referred from four catchment zones that includes three regional public and private hospitals. The outpatient clinic serves mainly discharged patients, for follow-up. Inclusion criteria were purposely broad to capture a representative sample of poorly adherent patients, and included a clinical diagnosis of schizophrenia or schizoaffective disorder, confirmed by a psychiatrist; self-reported poor medication adherence, defined as missing $\geq 20 \%$ of prescribed antipsychotic medication within the past week or past month; ${ }^{2}$ and being able to participate in assessment procedures and be rated on standardised rating scales. Individuals at risk of harm to themselves or others, and those who could not provide written informed consent to study participation, were excluded. The study was approved by the local institutional review board and the Tanzanian National Institute for Medical Research (ethical approval registry number: DA.282/298/01.C; ClinicalTrials.gov Identifier: NCT04327843).

\section{Assessments}

Evaluations comprised demographic and clinical characteristics relevant to CPD relapse. Demographics included age, gender, marital status, children/number of children, educational status, employment and where the individual resided (with family, alone, etc.). Clinical variables included type and duration of CPD, family psychiatric history, personal history of physical or sexual abuse, past hospital admissions and antipsychotic medication treatments.

Adherence with CPD medication in the past week and the past month (represented as proportion or percentage of days with a missed medication dose) was assessed with the Tablets Routine Questionnaire (TRQ). ${ }^{13,14}$ The TRQ ranges from $0 \%$ (missed no medications) to $100 \%$ (missed all medications). The ten-item Drug Attitudes Inventory (DAI) ${ }^{15}$ assessed attitudes toward CPD medications, with scoring calibrated on a 0 (worse attitudes) to 10 (best attitudes) spectrum. CPD symptoms were assessed with the Brief Psychiatric Rating Scale (BPRS). ${ }^{16}$ Global psychopathology was assessed with the Clinical Global Impressions scale (CGI). ${ }^{17}$ Life and work functional status was assessed with the Social and Occupational Functioning Scale (SOFAS). ${ }^{18}$ Substance use or misuse was assessed with the Alcohol Use Disorders Identification Test $(A U D I T)^{19}$ and the Alcohol, Smoking and Substance Involvement Screening Test (ASSIST). ${ }^{20}$ Although there is overlap between these two substance use instruments, the AUDIT specifically focuses on alcohol use patterns whereas the ASSIST evaluates a range of substances, including alcohol, tobacco, cannabis, cocaine, amphetamines, inhalants, sedatives and hallucinogens.

\section{Medication adherence barriers}

In preparation for a future phase of this research project involving pilot testing of an adherence promotion intervention, ${ }^{12}$ we assessed study participants on four main barriers to medication adherence: inadequate or incorrect understanding of CPD medication and consequences of missing medication, non-adherence related to substance use, limited communication with providers to address management of feared or experienced side-effects, and erratic medication-taking and/or lack of medication routines. The focus on these barriers and assessment process, drawn from iterative pilot work, ${ }^{5,21-23,40}$ assessed an individual's specific attitudinal adherence barriers with selected items from the Rating of Medication Influences (ROMI) ${ }^{24}$ and the Attitudes Toward Mood Stabilizers Questionnaire (AMSQ). ${ }^{25,26}$

\section{Data analysis}

We conducted descriptive statistics to characterise demographic and clinical variables, including the number of barriers to adherence. Because gender differences have been documented in treatment adherence and other variables among patients with $\mathrm{CPD},{ }^{27}$ we compared clinical characteristics of men and women in this sample, using chi-squared and two-tailed $t$-tests, reporting effect sizes. Correlational analyses with Spearman correlations (because of the non-normal distribution of lifetime hospital admissions) were conducted to evaluate the association between TRQ and demographic and clinical variables, as well as the relationship between lifetime number of hospital admissions for psychiatric illness and demographic and clinical variables. A multiple linear regression analysis was performed to assess the effects of in-patient versus out-patient status and DAI, BPRS, SOFAS and AUDIT scores on adherence over the past month, as measured by the TRQ.

\section{Results}

\section{Sample description}

Table 1 shows demographic and clinical variables in this poorly adherent sample. Notably, this was a relatively young sample (mean age 35.70 years, s.d. 8.80), with a slight preponderance of men (61\%). The majority (80\%) had schizophrenia, with an average age at onset of 22.38 (s.d. 7.64) years. The great majority of individuals lived with family (84\%) and just over half (57\%) had children. Most were in-patients (83\%) at the time of assessment, and had an average of 4.72 (4.30) previous hospital admissions for psychiatric illness. Most (>80\%) were prescribed first-generation antipsychotic drugs, with oral haloperidol being the most common medication therapy. Family history of mental illness and substance misuse were common, at rates of $53 \%$ and $43 \%$, respectively. Compared with men, women with CPD were less likely to live with family $(P<0.01, w=1.185)$, more likely to have a mood component to their CPD (schizoaffective diagnosis, $P=0.03, w=0.706$ ), had fewer lifetime hospital admissions for psychiatric illness $(P=$ $0.02, d=0.501)$ and had a greater likelihood of past sexual abuse $(P=0.01, w=0.602)$.

Table 2 shows scores on standardised rating scales in the sample. The proportion of missed CPD medication in the past week/month was extensive, with patients missing an average of nearly two-thirds of prescribed medication in the past month. CPD symptom scores were relatively low. Approximately threequarters of the sample were at low risk for having problems with substance misuse; however, one in ten had alcohol dependence, which was the most common substance of misuse. Substances other than alcohol and tobacco were not widely used. Based on the AMSQ and ROMI assessments, the most common adherence barrier domains were inadequate or incorrect understanding of the CPD and poor communication with providers. Approximately one in three had adherence barriers related to the use of substances.

\section{Adherence correlates}

Table 3 shows correlates of adherence over the past month, as measured by the TRQ. Demographic and most clinical variables were not significantly associated with TRQ levels; however, in-patients with CPD were more likely to have worse adherence $(r=-0.26$, $P \leq 0.01)$, as were individuals with worse drug attitudes $(r=$ $-0.28, P<0.01)$, higher CPD symptom severity levels $(r=0.36$, $P<0.001)$, worse functioning $(r=-0.21, P<0.05)$ and higher-risk use of alcohol (AUDIT score, $r=0.34, P<0.001$ ).

Using the five significant predictors at the bivariate level, we tested a multiple linear regression to determine which predictors 


\begin{tabular}{|c|c|c|c|c|}
\hline Variable & Mean (s.d.) or $n(\%), N=100$ & Male, $n=61$ & Female, $n=39$ & Statistic (d.f.) $)^{a}$ \\
\hline $\begin{array}{l}\text { Age, years } \\
\text { Marital status }\end{array}$ & $35.70(8.80)$, range $19-62$ & $36.26(9.28)$ & $34.82(8.04)$ & $t(98)=0.80, P=0.43$ \\
\hline Single, never married & $58(58.0 \%)$ & $38(62.3 \%)$ & 20 (51.3\%) & \multirow{3}{*}{$\chi^{2}(2)=2.44, P=0.30$} \\
\hline Married & $28(28.0 \%)$ & 17 (27.9\%) & $11(28.2 \%)$ & \\
\hline Separated/divorced/widowed & $14(14.0 \%)$ & $6(9.9 \%)$ & $8(20.5 \%)$ & \\
\hline \multicolumn{5}{|l|}{ Children $(n=99)$} \\
\hline Have children & 57 (57.0\%) & 30 (49.2\%) & $27(69.2 \%)$ & \multirow[t]{2}{*}{$\chi^{2}(1)=3.58, P=0.60$} \\
\hline Do not have children & $42(42.0 \%)$ & $30(49.2 \%)$ & $12(30.8 \%)$ & \\
\hline Number of children $(n=99)$ & 1.39 (1.81), range 0-10 & $1.18(1.71)$ & $1.72(1.93)$ & $t(97)=-1.44, P=0.15$ \\
\hline Education level, years $(n=99)$ & 9.42 (3.61), range $2-19$ & $9.00(3.44), n=60$ & $10.08(3.83)$ & $t(97)=-1.46, P=0.15$ \\
\hline \multicolumn{5}{|l|}{ Employment } \\
\hline Full time & $23(23.0 \%)$ & $10(16.4 \%)$ & $13(33.3 \%)$ & \multirow[t]{3}{*}{$\chi^{2}(2)=5.67, P=0.06$} \\
\hline Part time & $29(29.0 \%)$ & $22(36.1 \%)$ & 7 (17.9\%) & \\
\hline Unemployed & $48(48.0 \%)$ & $29(47.5 \%)$ & 19 (48.7\%) & \\
\hline \multicolumn{5}{|l|}{ Primary residence } \\
\hline Lives alone & $11(11.0 \%)$ & $5(8.2 \%)$ & $6(15.4 \%)$ & \multirow{3}{*}{$\chi^{2}(2)=10.07, P<0.01$} \\
\hline Lives with family & 84 (84.0\%) & 56 (91.8\%) & $28(71.8 \%)$ & \\
\hline Other & $5(5.0 \%)$ & $0(0.0 \%)$ & $5(12.8 \%)$ & \\
\hline \multicolumn{5}{|l|}{ Diagnosis } \\
\hline Schizophrenia & $80(80.0 \%)$ & 53 (86.9\%) & $27(69.2 \%)$ & \multirow[t]{2}{*}{$\chi^{2}(1)=4.63, P=0.03$} \\
\hline Schizoaffective & $20(20.0 \%)$ & $8(13.1 \%)$ & $12(30.8 \%)$ & \\
\hline Age at CPD onset & 22.38 (7.64), range 1-49 & $21.82(7.65)$ & $23.26(7.63)$ & $t(98)=-0.92, P=0.36$ \\
\hline Illness duration, years & 12.39 (7.99), range $1-36$ & $13.46(8.30)$ & $10.72(7.28)$ & $t(98)=1.69, P=0.10$ \\
\hline \multicolumn{5}{|l|}{ Treatment status at assessment } \\
\hline In-patient & 83 (83.0\%) & 52 (85.2\%) & 31 (79.5\%) & \multirow[t]{2}{*}{ Fisher's exact test, $P=0.59$} \\
\hline Out-patient & $17(17.0 \%)$ & $9(14.8 \%)$ & $8(20.5 \%)$ & \\
\hline \multicolumn{5}{|l|}{ CPD medication prescribed } \\
\hline Chlorpromazine & $8(8.0 \%)$ & $5(8.2 \%)$ & $3(7.7 \%)$ & \multirow[t]{5}{*}{$\chi^{2}(4)=2.42, P=0.66$} \\
\hline Haloperidol & 72 (72.0\%) & $46(75.4 \%)$ & 26 (66.7\%) & \\
\hline Olanzapine & $11(11.0 \%)$ & $6(9.9 \%)$ & $5(12.8 \%)$ & \\
\hline Risperidone & $8(8.0 \%)$ & $4(6.6 \%)$ & $4(10.3 \%)$ & \\
\hline Trifluoperazine & $1(1.0 \%)$ & $0(0.0 \%)$ & $1(2.6 \%)$ & \\
\hline \multicolumn{5}{|l|}{ Lifetime hospital admissions } \\
\hline Psychiatric & $4.72(4.30)$, median 3.00 & 5.49 (4.99) & $3.51(2.50)$ & $t(98)=2.30, P=0.02$ \\
\hline Substance misuse & 0.45 (1.35), median 0.00 & $0.64(1.64)$ & $0.15(0.59)$ & $\begin{array}{l}t(98)=2.30, P=0.02 \\
t(98)=1.77, P=0.08\end{array}$ \\
\hline \multicolumn{5}{|l|}{ History of abuse } \\
\hline Physical $(n=99)$ & & & & \\
\hline Yes & 55 (55.0\%) & $32(52.5 \%)$ & $23(59.0 \%)$ & \multirow{2}{*}{$\chi^{2}(1)=0.31, P=0.58$} \\
\hline No & 44 (44.0\%) & $28(45.9 \%)$ & $16(41.0 \%)$ & \\
\hline \multicolumn{5}{|l|}{ Sexual } \\
\hline Yes & 27 (27.0\%) & $11(18.0 \%)$ & $16(41.0 \%)$ & \multirow[t]{2}{*}{$\chi^{2}(1)=6.38, P=0.01$} \\
\hline No & $73(73.0 \%)$ & 50 (82.0\%) & $23(59.0 \%)$ & \\
\hline \multicolumn{5}{|l|}{ Family history } \\
\hline Mental illness & & & & \\
\hline Yes & 53 (53.0\%) & 34 (55.7\%) & 19 (48.7\%) & \multirow[t]{2}{*}{$\chi^{2}(1)=0.47, P=0.49$} \\
\hline No & 47 (47.0\%) & 27 (44.3\%) & 20 (51.3\%) & \\
\hline Alcohol misuse & & & & \\
\hline Yes & $43(43.0 \%)$ & $26(63.4 \%)$ & 17 (43.6\%) & $\chi^{2}(1)=0.01, P=0.92$ \\
\hline No & 57 (57.0\%) & 35 (57.4\%) & $22(56.4 \%)$ & \\
\hline
\end{tabular}

contributed the most to adherence. In-patient status (standardised $\beta=-0.219$, unstandardised $\beta=-21.872$, s.e. $0.088, \quad P<0.05$ ), worse symptom severity (standardised $\beta=0.211$, unstandardised $\beta=-1.529$, s.e. $0.103, P<0.05$ ) and higher-risk use of alcohol (standardised $\beta=0.241$, unstandardised $\beta=1.130$, s.e. $0.092, P<0.05$ ) were found to be associated with higher TRQ scores for adherence over the past month. DAI and SOFAS scores were not significant. The model explained $26.1 \%$ of the variance in adherence.

\section{Hospital admission correlates}

Tables 4 and 5 show correlates of lifetime number of hospital admissions for demographic/clinical variables and standardised ratings, respectively. As seen in Table 4 , males $(P=0.038)$, those with an earlier age at onset $(P=0.049)$, longer illness duration $(P<0.001)$ and those with children $(P=0.047)$ had significantly more hospital admissions for psychiatric illness. BPRS, CGI, AUDIT, DAI and TRQ scores were not associated with number of lifetime hospital admissions. Table 5 shows results of the multiple linear regression analysis assessing the effects of in-patient versus out-patient status and DAI, BPRS, SOFAS and AUDIT scores on adherence over the past month, as measured by the TRQ. In the linear regression analysis, there was a significant association between adherence and in-patient status, BPRS and AUDIT scores.

\section{Discussion}

The cross-sectional assessment of poorly adherent patients with CPD characterised demographic and clinical variables associated with medication adherence. It must be noted that the sample was specifically enrolled because they were known to be poorly 


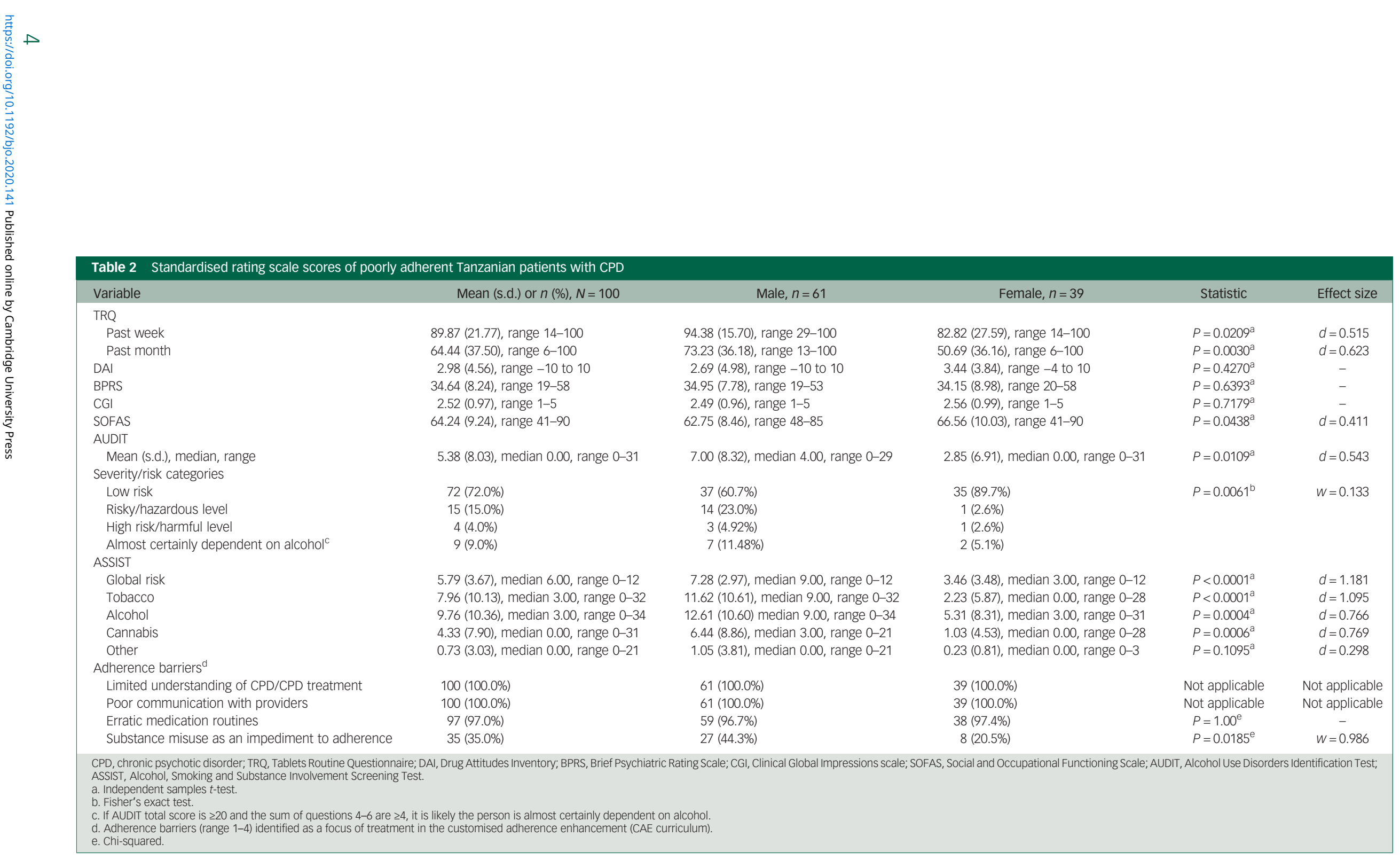


Table 3 Spearman correlations between adherence over the past month (measured by TRQ), demographics and standardised rating scales

\section{Variable}

$r_{\mathrm{s}}(P$-value $)$

\section{Age}

$0.01(0.34)$

Married versus not married ${ }^{a}$

Children versus do not have children $(n=99)^{\mathrm{b}}$

Number of children $(n=99)^{\mathrm{b}}$

Educational level $(n=99)^{b}$

Employed versus unemployed

Lives alone versus with others

schizophrenia versus schizoaffective

Age at onset

Illness duration

In-patient versus out-patient ${ }^{c}$

Hospital admissions for psychiatric illness

Substance misuse hospital admissions

Physical abuse versus none $(n=99)^{\text {b }}$

Sexual abuse versus none

Family mental illness versus none

Family alcohol misuse versus none

On haloperidol versus other medication

DAl ${ }^{\mathrm{c}}$

BPRS $^{\mathrm{C}}$

CGI-Global

SOFAS ${ }^{\mathrm{C}}$

AUDIT

$-0.01(0.89)$

$0.01(0.92)$

$0.13(0.22)$

$-0.04(0.72)$

$0.08(0.46)$

$-0.11(0.26)$

$0.03(0.77)$

$-0.13(0.20)$

$-0.26(<0.01)$

$-0.18(0.07)$

$0.04(0.71)$

$-0.06(0.58)$

$0.05(0.63)$

$-0.06(0.57)$

$0.01(0.95)$

$-0.06(0.55)$

$-0.28(<0.01)$

$0.36(<0.001)$

$0.04(0.70)$

$-0.21(0.04)$

$0.34(<0.001)$

TRQ, Tablets Routine Questionnaire; DAI, Drug Attitudes Inventory; BPRS, Brief Psychiatric Rating Scale; CGI, Clinical Global Impressions scale; SOFAS, Social and Occupational Functioning Scale; AUDIT, Alcohol Use Disorders Identification Test. a. Marital status categories were collapsed such that 'not married' included those who were single/never married as well as those who were separated, divorced or widowed. b. Ninety-nine participants completed the survey.

c. In-patient status is significantly correlated with a higher (worse) TRQ, more negative c. In-patient status is significantly correlated with a higher (worse) TRQ, more negative medication attitudes (lower DAl score) are associated with higher (worse) TRQ, mo severe psychiatric symptoms are associated with higher (worse) TRQ, worse (lower) social functioning is associated with higher (worse) TRQ, more substance use problems are associated with higher (worse) TRQ.

adherent, and findings may not generalise to the broader population of people with CPD in this setting, including those who are adherent with prescribed medications. For the most part, and aligned with reports from settings outside of sub-Saharan Africa, ${ }^{6}$ a number of demographic and clinical variables were not significantly associated with adherence levels; however, those with the worst adherence were most likely to be in-patients with more severe CPD symptoms, have substance use comorbidity and endorse negative attitudes toward CPD medications. Most individuals appear to have multiple barriers to adherence, including attitudinal characteristic that may be a potential target of future interventions.

Table 4 Spearman correlations between demographic variables and number of lifetime hospital admissions for psychiatric illness $(N=100)$

\section{Variable}

$r_{\mathrm{s}}(P$-value $)$

\section{Age}

Male (versus female)

Educational leve

Earlier age at onset (versus later)

Longer illness duration (versus shorter)

Individuals with children (versus without children)

History of physical abuse (versus no abuse)

History of sexual abuse (veruss no abuse)

Married $^{\text {a }}$ (versus not married)

Family history of mental illness (versus none)

Family history of alcohol misuse (versus none)

Employed (versus not employed)

$0.19(0.06)$

$-0.21(0.038)$

$-0.07(0.48)$

$-0.20(0.049)$

$0.38(<0.001)$

$-0.20(0.047)$

$-0.16(0.11)$

$-0.12(0.24)$

$0.06(0.52)$

$0.04(0.70)$

$0.08(0.46)$

$-0.01(0.94)$

$-0.01(0.93)$

Lives alone ${ }^{a}$ (versus lives with family)
Table 5 Associations from multiple linear regression models for past month medication adherence (TRQ)

\begin{tabular}{|c|c|c|c|c|c|}
\hline & $\begin{array}{c}\text { Unstandardised } \\
\beta \beta\end{array}$ & s.e. & $\begin{array}{c}\text { Standardised } \\
\beta\end{array}$ & s.e. & $P$-value \\
\hline $\begin{array}{c}\text { In-patient } \\
\text { versus } \\
\text { out- } \\
\text { patient }\end{array}$ & -21.872 & 9.25 & -0.219 & 0.09 & 0.020 \\
\hline DAl & -1.529 & 0.77 & -0.185 & 0.10 & 0.055 \\
\hline BPRS & 0.966 & 0.47 & 0.211 & 0.10 & 0.043 \\
\hline SOFAS & -0.082 & 0.41 & -0.020 & 0.10 & 0.842 \\
\hline AUDIT & 1.130 & 0.43 & 0.241 & 0.09 & 0.012 \\
\hline
\end{tabular}

Reasons for poor adherence in Tanzania include low health literacy, lack of access to medicines and structural factors (e.g. long distances to clinics to collect medicines, clinic overcrowding and low proportion of staff to patients). Similar to our sample, a review by Chidarikire $^{1}$ noted that the majority of people with schizophrenia were treated with first-generation antipsychotics that may cause motor and neurological side-effects, which are an impediment to adherence. $^{28-30}$ Although this analysis did not quantitatively assess for medication side-effects or medication preferences, recent qualitative work conducted by this team on a subset of patients in this sample found that burdensome side-effects with prescribed antipsychotic drugs included weight gain, weakness/tiredness and loss of libido, ${ }^{31}$ all negatively affecting treatment engagement and adherence. Accommodating patient preferences for specific treatments may be particularly challenging in settings where fewer types of medication options might be practical and available.

The average rate of missed CPD medication in this Tanzanian sample was $64 \%$, with an average of four or five past hospital admissions for psychiatric illness in just over a decade of having CPD. Other reports have documented the close association between poor adherence and poor outcomes, including relapse, leading to hospital admission and treatment-resistant illness. ${ }^{32,33}$ The findings from this sample suggest that most poorly adherent patients experience barriers to adherence in multiple domains, and it seems likely that care approaches will need to target patient attitudes/beliefs about medication, communication with providers to address treatment expectations and discussions regarding side-effects, and managing medication in the context of lifestyle and routines.

The in-patient setting may be an appropriate place to identify individuals at highest risk of poor adherence, and this might be an opportunity to provide education on the role of CPD medication in recovery. This may also be an opportunity to implement longacting injectable antipsychotic medication for individuals who have a known history of poor adherence or those who miss medication as a result of forgetting. Recent studies have suggested that long-acting injectable antipsychotic medication can significantly reduce relapse rates among individuals with schizophrenia. ${ }^{34}$

Adherence promotion approaches in sub-Saharan Africa and elsewhere also need to consider comorbidity as an impediment to optimal medication adherence. At least one in ten poorly adherent individuals with CPD in our sample had alcohol dependence, and about one in three had clinically relevant problems with use/misuse of substances. Thus, adherence and recovery efforts ideally need to include treatment for substance use disorders as part of a comprehensive package of care.

Our analysis found gender differences in selected clinical variables, including health resource use, with men more likely to be admitted to hospital. Differences in premorbid or early illness progression, also reported in the literature, might explain the fact that 
men had more lifetime hospital admissions compared with women. ${ }^{35,36}$ It is possible that addressing treatment adherence soon after CPD diagnosis might help with reducing hospital admissions, a particularly important goal in low-resource settings.

An additional factor that needs to be considered is the incorporation of family challenges and support in helping patients to self-manage treatment and adherence. The overwhelming majority (84\%) of individuals in this sample lived with family, and more than half had children of their own. Substance misuse and family history of both mental illness and substance misuse were common, and a pragmatic consideration would be to address the issue of mental health from an intergenerational perspective. Although our study did not find any correlation between living with family or living alone and treatment non-adherence, a Tanzanian qualitative study conducted by Sariah et $\mathrm{al}^{10}$ suggested that family and peer support, employment and religion were perceived as 'protective' elements to minimise patient risk of relapse episodes. Family therapies, including psychoeducation, have been demonstrated as improving outcomes and family burden in CPD, ${ }^{37-40}$ and culturally informed approaches could be helpful as an augmentation to adherencepromotion interventions.

This study has a number of methodological limitations that must be considered with respect to both interpretation and generalisability of findings. We did not survey an adherent group of patients, and findings on poorly adherent patients may not apply to those across the full range of adherence. Given the severe paucity of data on antipsychotic drug adherence in sub-Saharan African populations with $\mathrm{CPD}$, this study was exploratory and not designed or intended to be powered to examine gender differences in CPD or the specific variables that might be associated with worse adherence, such as substance use or personal trauma history. Given this exploratory nature, we did not control for multiple comparisons, and the cross-sectional design does not permit for conclusion of causal inferences. The study methods also do not permit a clear assessment of structural barriers to adherence, which are noted in the review by Chidarikire et $\mathrm{al}^{1}$ to include poor access to care as a result of poverty or other factors, lack of education or poor health literacy and problems in patient-clinician communication. These factors need to be more specifically addressed in future investigations.

Martha Sajatovic $\mathbb{D}, \mathrm{MD}$, Department of Psychiatry, Department of Neurology, Case Western Reserve University School of Medicine, University Hospitals Cleveland Medica Center, Ohio, USA; Jessie Mbwambo, MD, Department of Psychiatry and Mental Health School of Medicine, Muhimbili University of Health and Allied Sciences, Tanzania; Isaac Lema, PhD, Department of Psychiatry and Mental Health, School of Medicine, Muhimbili University of Health and Allied Sciences, Tanzania; Carol Blixen, PhD, Department of Psychiatry and Neurological and Behavioral Outcomes Center, Case Western Reserve University School of Medicine, Ohio, USA; Michelle E. Aebi, MA, Department of Psychiatry, Case Western Reserve University School of Medicine and University Hospitals Cleveland Medical Center, Ohio, USA; Betsy Wilson, MPH Department of Psychiatry, Case Western Reserve University School of Medicine and Department of Psychiatry, Case Western Reserve University School of Medicine
University Hospitals Cleveland Medical Center, Ohio, USA; Godwin Njiro, MD, University Hospitals Cleveland Medical Center, Ohio, USA; Godwin Njiro, MD,
Department of Psychiatry and Mental Health, School of Medicine, Muhimbili University of Health and Allied Sciences, Tanzania; Christopher J. Burant, PhD, Frances Payne Bolton School of Nursing, Case Western Reserve University, Ohio, USA; Kristin A. Cassidy, MA, Department of Psychiatry, Case Western Reserve University School of Medicine and University Hospitals Cleveland Medical Center, Ohio, USA Jennife

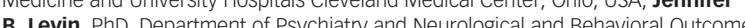
B. Levin, PhD, Department of Psychiatry and Neurological and Behavioral Outcomes Center, Case Western Reserve University School of Medicine and University Hospitals
Cleveland Medical Center, Ohio, USA; ; Sylvia Kaaya, MD, Department of Psychiatry and Mental Health, School of Medicine, Muhimbili University of Health and Allied Sciences, Tanzania

Correspondence: Martha Sajatovic. Email: martha.sajatovic@uhhospitals.org

First received 16 Mar 2020, final revision 2 Nov 2020, accepted 2 Nov 2020

\section{Supplementary material}

To view supplementary material for this article, please visit https://doi.org/10.1192/bjo.2020.141

\section{Data availability}

Scientists interested in accessing the data should reach out to the lead author (M.S.). Data access is subject to national USA and Ugandan data use agreement terms.

\section{Author contributions}

M.S., S.K. J.M. C.B., J.L, IL and K.A.C. contributed to either the initial design or refinement of the project design, as well as project implementation and completion. C.J.B. M.E.A. B.W. and G.N. contributed to project implementation, completion and data analysis. All authors have approved the manuscript, and the report is a reflection of team consensus.

\section{Funding}

This study was supported by the National Institute of Mental Health (NIMH) grant NIMH $\mathrm{MH} 114700$.

\section{Declaration of interest}

M.S. has received research grants from Otsuka, Alkermes, Janssen, Reuter Foundation, Woodruff Foundation, Reinberger Foundation, National Institutes of Health, Centers for Disease Control and Prevention and International Society of Bipolar Disorders, in the past 3 years; is a consultant for Bracket, Otsuka, Sunovion, Neurocrine, Supernus and Health Analytics; has received royalties from Springer Press, Johns Hopkins University Press, Oxford Press and UpToDate; and has participated in Continuing Medical Education activities with American Physician's Institute MCM Education, CMEology, Potomac Center for Medica Education, Global Medical Education and Creative Educational Concepts. All other authors Education, Global Medica

ICMJE forms are in the supplementary material, available online at https://doi.org/10.1192/ bjo.2020.141.

\section{References}

1 Chidarikire S, Cross M, Skinner I, Cleary M. Treatments for people living with schizophrenia in Sub-Saharan Africa: an adapted realist review. Int Nurs Rev 2018; 65: 78-92.

2 Velligan DI, Weiden PJ, Sajatovic M, Scott J, Carpenter D, Ross R, et al. Strategies for addressing adherence problems in patients with serious and persistent mental illness: recommendations from the expert consensus guidelines. J Psychiatr Pract 2010; 16: 306-24.

3 Gilmer TP, Dolder CR, Lacro JP, Folsom DP, Lindamer L, Garcia P, et al. Adherence to treatment with antipsychotic medication and health care costs among Medicaid beneficiaries with schizophrenia. Am J Psychiatry 2004; 161: 692-9.

4 Sajatovic M, Dawson NV, Perzynski AT, Blixen CE, Bialko CS, McKibbin CL, et al. Best practices: optimizing care for people with serious mental illness and comorbid diabetes. Psychiatr Serv 2011; 62: 1001-3.

5 Jenkins $\mathrm{JH}$, Strauss ME, Carpenter EA, Miller D, Floersch J, Sajatovic M. Subjective experience of recovery from schizophrenia-related disorders and atypical antipsychotics. Int J Soc Psychiatry 2005; 51: 211-27.

6 Semahegn A, Torpey K, Manu A, Assefa N, Tesfaye G, Ankomah A. Psychotropic medication non-adherence and its associated factors among patients with major psychiatric disorders: a systematic review and meta-analysis. Syst Rev 2020; 9: 17.

7 Adewuya AO, Owoeye OA, Erinfolami AR, Coker AO, Ogun OC, Okewole AO, et al. Prevalence and correlates of poor medication adherence amongst psychiatric outpatients in Southwestern Nigeria. Gen Hosp Psychiatry 2009; 31: 167-74.

8 Adeponle AB, Thombs BD, Adelekan ML, Kirmayer LJ. Family participation in treatment, post-discharge appointment and medication adherence at a Nigerian psychiatric hospital. Br J Psychiatry 2009; 194: 86-7.

9 Danladi J, Falang KD, Barde RA, Jimam NS, Dangiwa DA, Jimoh HO. Pharmaceutical care and medication adherence in management of psychosis in a Nigerian tertiary hospital. J Res Pharm Pract 2013; 2: 83-7.

10 Sariah $\mathrm{AE}$, Outwater $\mathrm{AH}$, Malima $\mathrm{KI}$. Risk and protective factors for relapse among individuals with schizophrenia: a qualitative study in Dar es Salaam, Tanzania. BMC Psychiatry 2014; 14: 240.

11 Ho Odo OS, Agbonile IO, Esan PO. Assessment of adherence to psychotropic medications among outpatients at the pharmacy department of a psychiatric hospital in Benin City, Nigeria. Asian J Pharm 2014; 194: 86-7.

12 Mbwambo J, Kaaya S, Lema I, Blixen C, Cassidy KA, Levin JB, et al. A customized adherence enhancement program combined with long-acting injectable antipsychotic medication (CAE-L) for poorly adherent patients with chronic psychotic disorder in Tanzania: a pilot study methodological report. Heliyon 2019; 5(6): e01763.

13 Scott J, Pope M. Nonadherence with mood stabilizers: prevalence and predictors. J Clin Psychiatry 2002; 63: 384-90. 
14 Peet M, Harvey NS. Lithium maintenance: 1. A standard education programme for patients. Br J Psychiatry 1991; 158: 197-200.

15 Awad AG. Subjective response to neuroleptics in schizophrenia. Schizophr Bull 1993; 19: 609-18.

16 Overall JE, Gorham DR. The Brief Psychiatric Rating Scale. Psychol Rep 1962; 10 799-812.

17 Guy W. ECDEU Assessment Manual for Psychopharmacology. US Department of Health, Education, and Welfare, 1976.

18 Morosini PL, Magliano L, Brambilla L, Ugolini S, Pioli R. Development, reliability and acceptability of a new version of the DSM-IV Social and Occupational Functioning Assessment Scale (SOFAS) to assess routine social functioning. Acta Psychiatr Scand 2000; 101: 323-9.

19 Saunders JB, Aasland OG, Babor TF, Grant M, Fuente JR. Development of the Alcohol Use Disorders Identification Test (AUDIT): WHO collaborative project on early detection of persons with harmful alcohol consumption-II. Addiction 1993; 88: 791-804.

20 WHO ASSIST Working Group. The Alcohol, Smoking and Substance Involvement Screening Test: development, reliability, and feasibility. Addiction 2002; 97 : 1183-94.

21 Sajatovic M, Levin J, Ramirez LF, Hahn DY, Tatsuoka C, Bialko CS, et al. Prospective trial of customized adherence enhancement plus long-acting injectable antipsychotic medication in homeless or recently homeless individuals with schizophrenia or schizoaffective disorder. J Clin Psychiatry 2013; 74: $1249-55$

22 Sajatovic M, Levin J, Tatsuoka C, Micula-Gondek W, Williams TD, Bialko CS, et al. Customized adherence enhancement for individuals with bipolar disorder receiving antipsychotic therapy. Psychiatr Serv 2012; 63: 176-8.

23 Sajatovic M, Levin J, Tatsuoka C, Micula-Gondek W, Fuentes-Casiano E, Bialko $\mathrm{CS}$, et al. Six-month outcomes of customized adherence enhancement (CAE) therapy in bipolar disorder. Bipolar Disord 2012; 14: 291-300.

24 Weiden P, Rapkin B, Mott T, Zygmunt A, Goldman D, Horvitz-Lennon M, et al. Rating of medication influences (ROMI) scale in schizophrenia. Schizophr Bull 1994; 20: 297-310.

25 Harvey NS. The development and descriptive use of the Lithium Attitudes Questionnaire. J Affect Disord 1991; 22: 211-9.

26 Adams J, Scott J. Predicting medication adherence in severe mental disorders. Acta Psychiatr Scand 2000; 101: 119-24.

27 Abel KM, Drake R, Goldstein JM. Sex differences in schizophrenia. Int Rev Psychiatry 2010; 22: 417-28.
28 Esan O. Persistence in treatment for one year among patients in Nigeria with first-episode schizophrenia. Psychiatr Serv 2014; 65: 1174-6.

29 Kazadi NJB, Moosa MYH, Jeenah FY. Factors associated with relapse in schizophrenia. S Afr J Psychiatry 2008; 14: 52-62.

30 Van Rensburg AJ, Oloruniu S. Diagnosis and treatment of schizophrenia in a general hospital based acute psychiatric ward. Afr J Psychiatry 2010; 13: 204-10.

31 Blixen C, Lema I, Mbwambo J, Kaaya S, Levin JB, Sajatovic M. Community perceptions of barriers to management of chronic psychotic disorders and knowledge and attitudes about long-acting injectable antipsychotic medication: qualitative study in Dar es Salaam, Tanzania. BJPsych Open 2020; 6: e27.

32 Phan SV. Medication adherence in patients with schizophrenia. Int J Psychiatry Med 2016; 51: 211-9.

33 Bozzatello P, Bellino S, Rocca P. Predictive factors of treatment resistance in first episode of psychosis: a systematic review. Front Psychiatry 2019; 10: 67.

34 Tiihonen J, Mittendorfer-Rutz E, Majak M, et al. Real-world effectiveness of antipsychotic treatments in a nationwide cohort of 29,823 patients with schizophrenia. JAMA Psychiatry 2017; 74(7): 686-93.

35 Abel KM, Drake R, Goldstein JM. Sex differences in schizophrenia. Int Rev Psychiatry 2010; 22: 417-28.

36 Leung A, Chue P. Sex differences in schizophrenia, a review of the literature. Acta Psychiatr Scand Suppl 2000; 401: 3-38.

37 McFarlane WR. Family interventions for schizophrenia and the psychoses: a review. Fam Process 2016; 55: 460-82.

38 Mubin MF, Riwanto I, Soewadi SH, Erawati E. Psychoeducational therapy with families of paranoid schizophrenia patients. Enferm Clin 2020; 30(5): 326-32.

39 Weisman de Mamani A, Weintraub MJ, Gurak K, Maura J. A randomized clinical trial to test the efficacy of a family-focused, culturally informed therapy for schizophrenia. J Fam Psychol 2014; 28: 800-10.

40 Edge D, Degnan A, Cotterill S, Berry K, Baker J, Drake R, et al. Culturally Adapted Family Intervention (CaFI) for African-Caribbean People Diagnosed with Schizophrenia and their Families: A Mixed-Methods Feasibility Study of Development, Implementation and Acceptability. Health Services and Delivery Research, No. 6.32. NIHR Journals Library, 2018 (https://www.ncbi. nlm.nih.gov/books/NBK525363/).

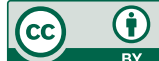

